\title{
EXTENDING DIFFERENTIAL SPECIALIZATIONS ${ }^{1}$
}

\section{PETER BLUM}

The study of specializations in differential algebra has been marked by the appearance of several examples which behave very differently from their less complicated brethren in algebraic geometry.

In fact, let $\mathfrak{F}$ denote an ordinary differential field of characteristic zero, $\mathfrak{U}$ a universal extension of $\mathfrak{F}$ with field of constants $K$, with $y$ and $z$ differential indeterminates over $\mathfrak{U}$ (these notational conventions will be observed from now on). Subscripts will denote derivatives. Then Kolchin has shown that if $A=y z_{1}^{2}+P(z)$, where $P$ is a cubic polynomial having distinct roots with coefficients in $\mathfrak{F} \cap K$, and if $(\eta, \zeta)$ is a generic zero of the general component of $A$ in $\mathfrak{F}\{y, z\}$ then 0 is a differential specialization of $\eta$ over $\mathfrak{F}$ but there is no element $\alpha \in \mathfrak{U}$ such that $(0, \alpha)$ is a differential specialization of $(\eta, \zeta)$ or $\left(\eta, \zeta^{-1}\right)$ over $\mathfrak{F}$.

Another example, which goes back to Ritt [3], shows the existence of another prime differential ideal in $\mathfrak{F}\{y, z\}$ whose generic zero $(\eta, \zeta)$ has the property that 0 is a differential specialization of $\eta \zeta$ over $\mathfrak{F}$, but not of $\eta$ or of $\zeta$ over $\mathfrak{F}$.

The situation arising out of the first example was dealt with in [1] where it was shown that if $R$ is a local differential domain, meaning that $R$ is local and has a maximal ideal $m$ which is differential, then if the differential homomorphism $\phi: R \rightarrow R / m$ cannot be extended either to $\alpha$ or to $\beta$, there exist nonnegative integers $i, j$ such that $\alpha_{i} \beta_{j} \notin R$. As a corollary it was shown that if $\alpha_{1} / \alpha \in R$ then $\phi$ can be extended either to a differential homomorphism of $R\{\alpha\}$ or to one of $R\{1 / \alpha\}$.

In the reference cited $\alpha$ and $\beta$ were restricted to the differential field of quotients of $R$, but it is easy to see that this assumption is not necessary. The present paper is concerned with the second of the above-mentioned phenomena and it will be shown that under a certain condition $\eta$ or $\zeta$ must specialize to 0 over $\mathfrak{F}$.

I would like to thank Professor Ellis Kolchin and Dr. Jerald Kovacic for their helpful thoughts on this subject.

DEFinition. Let $R$ be a differential subring of $\mathfrak{U}$ containing the rational numbers, $Q$. An element $\beta \in \mathfrak{U}$ is said to be monic over $R$ if $\beta$ is a zero of a differential polynomial of the form $y^{n}+f(y) \in R\{y\}$ where the total degree of $f$ is less than $n$.

Received by the editors April 11, 1969.

1 This research has been partially supported by the National Science Foundation under Grant NSF GP-8718. 
Proposition. Let $R, \beta$ be as above with $\beta \neq 0$, and $R$ a local differential ring. Let $m$ be the maximal ideal in $R$. Then $\beta$ is monic over $R$ if the differential homomorphism $R \rightarrow R / m$ cannot be extended to a differential homomorphism $\phi: R\{1 / \beta\} \rightarrow \mathfrak{U}$ with $\phi(1 / \beta)=0$. Conversely, if $\beta$ is monic over $R$, then no differential homomorphism $\phi: R \rightarrow \mathfrak{U}$ can be extended to one sending $1 / \beta$ into 0 ( $R$ need not be local here).

Proof. If the natural map $R \rightarrow R / m$ has no such extension, then the differential ideal in $R\{1 / \beta\}$ generated by $m$ and $1 / \beta$ is the unit ideal. Thus $1=m_{0}+\sum_{i} N_{i}(1 / \beta)$ where $m_{0} \in m$ and the $N_{i}$ are differential monomials with coefficients in $R$. Clearing fractions and dividing by $1-m_{0}$ we see that $\beta$ is monic over $R$.

For the converse, assume that $\beta$ is monic over $R$. Then $1 / \beta$ is a zero of a differential polynomial of the form $y^{r}+g(y) \in R\{y\}$ where $r$ is less than the degree of any monomial occurring in $g$ (set $\gamma=1 / \beta$ and clear fractions in the equation $(1 / \gamma)^{n}+f(1 / \gamma)=0$ satisfied by $\left.\beta\right)$.

Let $A\left(y, u_{i}\right)=y^{r}+g\left(y, u_{i}\right) \in Q\left\{y, u_{i}\right\}$ be the differential polynomial obtained from $y^{r}+g(y)$ by replacing the coefficients $r_{i}$ from $R-Q$ by differential indeterminates $u_{i}$. By Levi's Lemma [2, §32] there is a differential polynomial $1+Y$ where $Y \in[y] \subset Q\left\{y, u_{i}\right\}$ such that $y(1+Y) \in\{A\}$. Since $\{A\}$ is the intersection of prime differential ideals, one of them, say $P$, must vanish at the point $\left(1 / \beta, r_{i}\right)$. Since $y \notin P, 1+Y \in P$; but if $\tilde{\phi}$ were an extension of $\phi$ to $R\{1 / \beta\}$ with $\tilde{\phi}(1 / \beta)=0$ and $\psi: Q\{y, u\} \rightarrow \mathfrak{U}$ is defined by $\psi(y)=0, \psi\left(u_{i}\right)=r_{i}$ for every $i$, then $\psi(1+Y)=\left(\tilde{\phi} \circ \psi_{1}\right)(1+Y)$, where $\psi_{1}: Q\left\{y, u_{1}\right\} \rightarrow \mathfrak{U}$ is defined by $\psi_{1}(y)=1 / \beta, \psi_{1}\left(u_{i}\right)=r_{i}$ for all $i$. However this is impossible since $\psi(1+Y)=1$ and $\psi_{1}(1+Y)=0$.

REMARK. Let $\mathfrak{F}$ and $\mathfrak{U}$ be as in the introduction. There is an unpublished result of Seidenberg which states that if $\alpha \in \mathfrak{U}$ and if there is a differential specialization over $\mathfrak{F}$ with $\alpha \mapsto 0$ then this can be extended to $\left(\alpha, \alpha_{1} / \alpha\right) \mapsto(0, \gamma)$ over $\mathfrak{F}$, for some $\gamma \in \mathfrak{U}$.

This is also a consequence of the proposition for if $\phi: \mathfrak{F}\{\alpha\} \rightarrow \mathfrak{U}$ is a differential homomorphism with $\phi(\alpha)=0$ then $1 / \alpha$ is not monic over $R_{1}=\mathfrak{F}\{\alpha\}$ and hence $1 \notin[\alpha] R_{1}\left\{\alpha_{1} / \alpha\right\}$ since if $\beta=1 / \alpha$ and $1 \in[1 / \beta] R_{1}\left\{\beta_{1} / \beta\right\}$ then $1=f(\beta) / \beta^{N}$, where $f$ is a differential polynomial of degree less than $N$. This would imply that $\beta$ is monic over $R_{1}$, a contradiction.

Definition. If $R$ is a differential integral domain, $\phi: R \rightarrow \mathfrak{U}$ a differential homomorphism, then $\phi$ is called a differential place of the differential field of quotients $K$ of $R$ if $\phi$ cannot be properly extended to a differential homomorphism whose domain properly contains $R$ 
and is contained in $K . R$ is called the ring of $\phi$ and is easily seen to be a local differential ring.

THEOREM. Let $\mathfrak{F}$ be an ordinary differential field of characteristic zero, $\mathfrak{U}$ a universal extension of $\mathfrak{F}, \eta, \zeta \in \mathfrak{U}-\{0\}$. Suppose there exists a differential homomorphism $\phi_{0}: \mathfrak{F}\{\eta \zeta\} \rightarrow \mathfrak{U}$ over $\mathfrak{F}$ with $\phi_{0}(\eta \zeta)=0$. If $\phi_{0}$ can be extended to a differential place $\phi$ of $\mathfrak{F}\langle\eta \zeta\rangle$ over $\mathfrak{F}$ having ring $R$ with $(\eta / \zeta)_{1} / \eta / \zeta$ and $(\eta \zeta)_{1} / \eta \zeta \in R$, then $\phi_{0}$ can either be extended to a differential specialization with $\eta \mapsto 0$, or to one with $\zeta \mapsto 0$ over $\mathfrak{F}$.

PROOF. Since $\eta_{1} / \eta+\zeta_{1} / \zeta \in R$ and $\eta_{1} / \eta-\zeta_{1} / \zeta \in R$, we see that $\eta_{1} / \eta$ and $\zeta_{1} / \zeta \in R$. Hence there is a differential place of $\mathfrak{F}\langle\eta, \zeta\rangle$ whose ring $S$ contains $R$ and either $\eta$ or $1 / \eta$. Moreover, since $\zeta_{1} / \zeta \in S$ it follows that $\zeta$ or $1 / \zeta \in S$.

If the conclusion were false, then $(1 / \eta) \cdot(1 / \zeta) \in S$, contradicting the fact that $\eta \zeta$ belongs to the maximal ideal of $S$.

Corollary 1. Using the same notation as in the theorem, if $\eta_{1} / \eta$ $=s \zeta_{1} / \zeta$ where $1 /(s+1) \in R$, then $\phi_{0}$ can either be extended to a differential specialization with $\eta \mapsto 0$ or to one with $(\zeta) \mapsto 0$ over $\mathfrak{F}$.

Proof. Since $\eta_{1} / \eta+\zeta_{1} / \zeta=(s+1) \zeta_{1} / \zeta \in R$, one can repeat the above reasoning. However, it is worth noting that the condition on $\eta$ and $\zeta$ in the corollary implies that in the theorem.

Indeed if $\eta_{1} / \eta=s \zeta_{1} / \zeta$ then $\eta_{1} / \eta-\zeta_{1} / \zeta=a \in R$ and we can find a $b \in \mathfrak{U}$ such that $b_{1}=a b$. Then "integrate" $(\eta / \zeta)_{1} / \eta / \zeta=b_{1} / b$ to get $c \in K$ such that $r=c b$ works, where $\eta=r \zeta$.

Taking $\eta=\zeta$ in the theorem or in Corollary 1 we get the case $n=2$ of

Proposition 2. If $n$ is a positive integer and there exists a differential specialization with $\eta^{n} \mapsto 0$ over $\mathfrak{F}$, then there exists one with $\eta \mapsto 0$ over $\mathfrak{F}$.

Proof. There exists a differential place $\phi$ of $\mathfrak{F}\langle\eta\rangle$ whose ring $R$ contains $n \eta^{n-1} \eta_{1} / \eta^{n}$ and hence $\eta_{1} / \eta$. Therefore $\phi(\eta)=0$.

REMARKs. (1) If $\eta^{n \mapsto a}$ where $a$ is a nonzero element of $\mathfrak{F}$ then it is easy to show that $\eta$ can specialize simultaneously to a nonzero element of $\mathfrak{U}$ by using the fact that the differential value group of a differential place $\phi$ (that is, the nonzero elements of $\mathfrak{u}$ modulo the units of the ring $R$ of $\phi)$ is torsion free. See [1] for more details.

(2) Choosing indeterminates $y_{1}, \cdots y_{n}$ over $\mathfrak{U}$ Kolchin can show that if $\left(\eta_{1}^{l_{1}}, \cdots, \eta_{n}^{\ell_{n}}\right) \mapsto(0,0, \cdots 0)$ over $\mathfrak{F}$, where $l_{1}, \cdots, l_{n}$ are positive integers and subscripts do not denote derivatives, then $\left(\eta_{1}, \cdots, \eta_{n}\right) \mapsto(0,0, \cdots 0)$ over $\mathfrak{F}$. His argument is in the context of partial differential algebra. 
Corollary 2. Assume that $\mathfrak{F}$ contains the field of complex numbers. If $\eta \in \mathfrak{U}, n$ a positive integer and $c \in K$ with $\eta^{n} c \mapsto 1$ over $\mathfrak{F}$, then either $(\eta, 1 / c) \mapsto(0,0)$ over $\mathfrak{F}$ or there exist $\alpha, \beta \in \mathfrak{U}$ with $(1 / \eta, c) \mapsto(\alpha, \beta)$ over $\mathfrak{F}$. Moreover $\alpha=0$ if and only if $\beta=0$.

Proof. Suppose $n=1$. We have $(1): \eta(1 / \eta-c) \mapsto 0$ over $\mathfrak{F}$ and if we let $\zeta=-1 / \eta+c$ then $\zeta_{1}=\eta_{1} \eta^{-2}$ and hence $\eta_{1} / \eta=(\eta \zeta) \zeta_{1} / \zeta$. It follows from Corollary 1 that the differential specialization (1) can be extended either to $\eta \mapsto 0$ or $1 / \eta-c \mapsto 0$ over $\mathfrak{F}$. In the first case we can extend to $1 / c \mapsto 0$ over $\mathfrak{F}$ since $c_{1} / c=0$ and there is clearly no extension to $c$.

On the other hand, if $\eta \nrightarrow 0$ then $1 / \eta$ and $1 / \eta-c$ belong to $S$ and the maximal ideal of $S$, respectively, where $S$ is the ring of a differential place $\phi$ of $\mathfrak{F}\langle\eta, \zeta\rangle$, as in the proof of the theorem. Let $\alpha=\phi(1 / \eta)$ and $\beta=\phi(c)$.

In general, $\left(\eta c^{1 / n}\right)^{n \mapsto 1}$ over $\mathfrak{F}$ and by the first remark, $\eta c^{1 / n \mapsto d}$ for some $d$ with $d^{n}=1$. Hence $\left(\eta, c^{-1 / n} d\right) \mapsto(0,0)$ or $\left(1 / \eta, c^{1 / n} \cdot 1 / d\right) \mapsto(\alpha, \gamma)$ over $\mathfrak{F}$.

In the first case $1 / c \mapsto 0$ as required, while in the second $c \mapsto \gamma^{n}=\beta$.

Finally, we consider the necessity of the condition on $\eta / \zeta$ in the theorem. Using the notation therein, suppose $\phi_{0}$ can be extended to a differential specialization $\eta \mapsto 0$, say. Then this can be further extended to one of $\eta_{1} / \eta$. If $\eta_{1} / \eta \in R$ then $\eta_{1} / \eta-\zeta_{1} / \zeta=a \in R$ and we proceed as in Corollary 1 to find $r \in \mathfrak{U}$ with $\eta=r \zeta$ and $r_{1} / r \in R$.

In general we do not know whether the condition is necessary.

\section{REFERENCES}

1. Peter Blum, Complete models of differential fields, Trans. Amer. Math. Soc. 137 (1969), 309-325.

2. Howard Levi, On the structure of differential polynomials and on their theory of ideas, Trans. Amer. Math. Soc. 51 (1942), 532-568. MR 3, 264.

3. J. F. Ritt, On a type of algebraic differential manifold, Trans. Amer. Math. Soc. 48 (1940), 542-552. MR 2, 197.

Columbia University 